University of New Orleans

ScholarWorks@UNO

Physics Faculty Publications

Department of Physics

1997

\title{
Intrinsic chaos in a dc field biased quantum heterostructure
}

\author{
A. Jason McNary \\ University of New Orleans \\ Ashok Puri \\ University of New Orleans
}

Follow this and additional works at: https://scholarworks.uno.edu/phys_facpubs

Part of the Physics Commons

\section{Recommended Citation}

J. Appl. Phys. 82, 1687 (1997)

This Article is brought to you for free and open access by the Department of Physics at ScholarWorks@UNO. It has been accepted for inclusion in Physics Faculty Publications by an authorized administrator of ScholarWorks@UNO.

For more information, please contact scholarworks@uno.edu. 


\title{
Intrinsic chaos in a dc field biased quantum heterostructure
}

\author{
A. Jason McNary a) and Ashok Puri \\ Department of Physics, University of New Orleans, New Orleans, Louisiana 70148
}

(Received 17 July 1996; accepted for publication 23 May 1997)

\begin{abstract}
A closed, quantum, double barrier, GaAs/AlGaAs heterostructure is made chaotic by adding a nonlinear potential term, $\alpha\langle Q(t)\rangle$, to the time-dependent Schrödinger equation, and the dynamical behavior of an electron cloud moving in the heterostructure biased by a dc electric field is examined numerically. Using phase-space diagrams, power spectrums, and Lyapunov exponents, both qualitative and quantitative measures of the chaos in the system were taken. In general, for all values of $\alpha$, the nonlinearity parameter, the Lyapunov exponent, $\lambda$, increases as the applied dc field, $\beta$, increases. However, for values of $\alpha \leqslant 1.376$, we notice a sharp drop in $\lambda$ for the value of $\beta$ $=-9.2 \times 10^{7} \mathrm{~V} / \mathrm{m}$ corresponding to an average dc voltage of $-.085 \mathrm{eV}$ in the central well. This first order type transition to high values of $\lambda$ for $\alpha>1.376$ corresponds to a similar increase in the mean charge trapped in the heterostructure and in the average nonlinear potential in the central well for that $\mathrm{dc}$ field. This behavior is attributed to the fact that for $\alpha \leqslant 1.376$ and $\beta=-9.2$ $\times 10^{7} \mathrm{~V} / \mathrm{m}$, the field effects dominate, but for $\alpha>1.376$, the nonlinearity term dominates. (C) 1997 American Institute of Physics. [S0021-8979(97)07616-0]
\end{abstract}

\section{INTRODUCTION}

Multiple barrier heterostructures are rapidly gaining popularity in device applications today. Among the many uses are frequency multipliers, multistable memory, diode lasers, and high speed analog to digital converters. ${ }^{1}$ One of the most useful heterostructures is the $\mathrm{GaAs} / \mathrm{Ga}_{1-x} \mathrm{Al}_{x} \mathrm{As}$ heterostructure where the GaAs layer has a narrower band gap than the $\mathrm{Ga}_{1-x} \mathrm{Al}_{x} \mathrm{As}$. Currently, it is possible to fabricate structures with thicknesses of $7.5 \AA$ for layers of GaAs. ${ }^{2}$ With such minute widths, quantum devices are becoming commonplace. For example, Fowler and Datta et al. have both independently proposed a quantum interference transistor. ${ }^{3}$

In resonant tunneling structures, such as transistors and diodes, it is necessary to apply an external electric field to bias the structure properly. One of the major reasons for this is that the application of a strong electric field reduces the characteristic time $\tau=\hbar / \partial E$ of the structure with $\partial E$ being the width of the transmission probability for the linear case. With the appropriate choice of the external electric field, one can cut the characteristic time of the system in half. ${ }^{4}$ The increase in the operating frequency of the heterostructure makes it more desirable for devices. Thus the electric field is a very important parameter in quantum devices.

In previous work, it has been noted that an unbiased double barrier potential well in a closed system exhibits chaos as an intrinsic property of the well. ${ }^{5,6}$ Charge accumulates in the well created by the two barriers and creates a self-consistent potential that leads to the nonlinearity in the system. The transient behavior of the charge trapped in the well has been shown to be chaotic with the chaos increasing as the nonlinearity increases. ${ }^{5}$ Because heterostructures must have an electric field applied to be used in device applications, it is even more important to study the behavior of the

\footnotetext{
a) Present address: Department of Physics, University of California, Riverside, CA 92507.
}

trapped charge in biased structures. This article will examine the chaotic characteristics of GaAs/AlGaAs structures for several applied dc fields for different strengths of the nonlinearity in the well. The phase-space diagram and power spectrum will be examined as qualitative indicators along with the Lyapunov exponent for a quantitative analysis of the system.

\section{MODEL SYSTEM}

Figure 1(a) depicts our model system's potential profile which is a traditional double-barrier heterostructure contained by infinite barriers at both ends. Upon applying a dc bias to only the inner well, the barrier shifts down, allowing for easier tunneling in one direction as shown in Fig. 1(b). In our model, a cloud of electrons propagates in the biased heterostructure potential profile, and the motion of the cloud is approximated as a mean field using a Hartree-like equation. The incoming electrons to the central well region are subjected to a repulsive mean field potential arising from the charge build-up in the well, $w_{2}$. Thus the complete potential, as shown in Fig. 1, consists of a self-consistent, time dependent, nonlinear term in the central well. The nonlinearity creates a nondissipative system that mixes in some regions of its phase space. This causes the system to exhibit chaotic behavior during its evolution as has been shown in the unbiased case. ${ }^{5}$

A packet of electrons is created in the wide well, $w_{1}$, and it is launched towards the central well. The charge trapped by resonance in the central well generates a reaction field which modifies the time evolution of the system. We assume a decoupling of longitudinal and transverse degrees of freedom of the system. This is a common assumption made in tunneling phenomena, and it allows us to treat the problem as one dimensional, easing the calculations in the problem. ${ }^{4-6}$ 
(a)

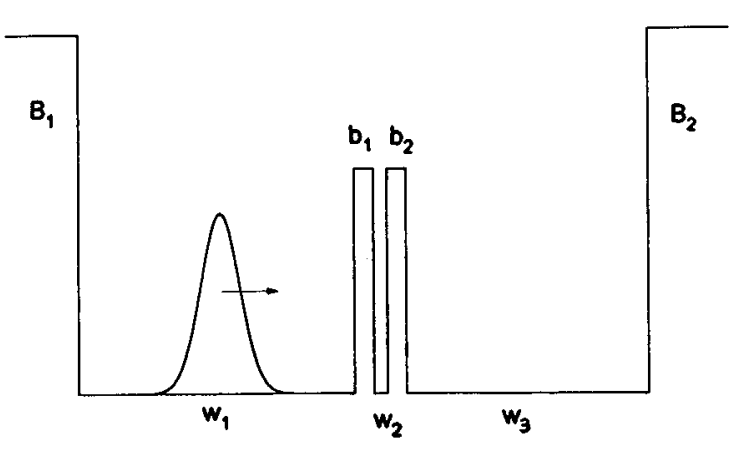

(b)

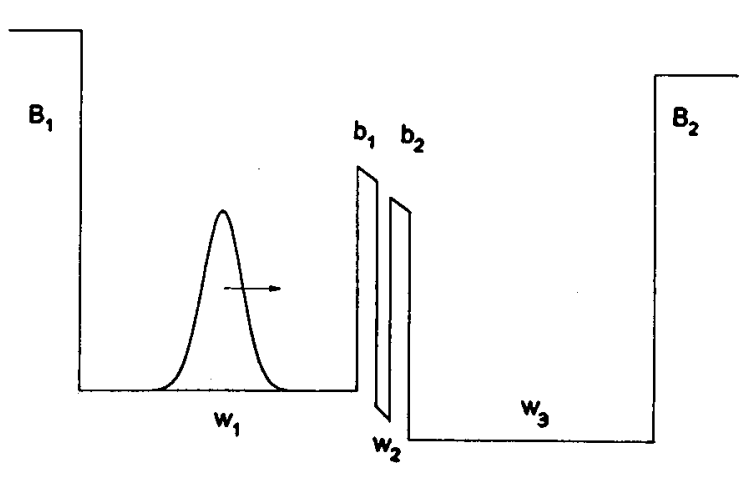

FIG. 1. Model potential structure of a three-well, double-barrier heterostructure for (a) the unbiased case and (b) the dc biased case.

We used the same model system as Rosa et al., ${ }^{4}$ and the mathematical description of the unbiased structure is given by

$$
\begin{aligned}
i \hbar \frac{\partial \Psi(x, t)}{\partial t}= & \frac{-\hbar^{2}}{2 m} \frac{\partial^{2} \Psi(x, t)}{\partial x^{2}}+[V(x) \\
& \left.+\alpha Q(t) \chi_{w_{z}}\right] \Psi(x, t) .
\end{aligned}
$$

$V(x)$ is the potential profile of the heterostructure. $\chi$ is a theta function with $\chi_{w_{2}}=1$ inside the well, $w_{2}$, and $\chi_{w_{2}}$ $=0$ elsewhere. The nonlinear term $Q(t)$ is defined by

$$
Q(t)=\int_{w_{2}}|\Psi(x, t)|^{2} d x
$$

and it is weighted by a nonlinear coefficient, $\alpha$. This allows us to adjust the strength of the nonlinearity without adjusting the shape of our potential profile. The nonlinear coefficient, $\alpha$, is inversely proportional to capacitance per unit area and directly proportional to the areal number density of the incident electrons, i.e., the nonlinear coupling will be greater if a greater number of electrons are incident. Thus

$$
\alpha \propto \frac{e\left(n_{s}\right)}{C},
$$

where $e$ is the electron charge, $n_{s}$ is the areal number density of the incident electrons, and $C$ is the capacitance per unit area of the heterostructure. The nonlinearity parameter, $\alpha$, can be varied to reproduce phenomenologically the response of the medium to the charge trapped in the well and to the characteristics of the incident group of electrons (the areal density). $n_{s}$ can be varied by changing the doping levels in the heterostructure. By this method, $\alpha$ can be controlled experimentally.

The potential profile $V(x)$ is considered for two different cases. In the first, there is no external field applied [Fig. 1(a)],

$$
V(x)=V_{0}\left[\chi_{b_{1}}(x)+\chi_{b_{2}}(x)\right]+V_{1}\left[\chi_{B_{1}}(x)+\chi_{B_{2}}(x)\right],
$$

where $V_{0}$ and $V_{1}$ are positive constants (i.e., the height of barriers $b_{1}, b_{2}$, and $\left.B_{1}, B_{2}\right)$. This is the structure that is investigated in Ref. 5. When the electric field is applied, the profile takes on the shape given by [Fig. 1(b)]

$$
\begin{aligned}
V(x)= & V_{0}\left[\chi_{b_{1}}(x)+\chi_{b_{2}}(x)\right]+V_{1}\left[\chi_{B_{1}}(x)+\chi_{B_{2}}(x)\right] \\
& +V_{2} \chi_{b_{1}, w_{2}, b_{2}}(x)+V_{3} \chi_{w_{3}}(x),
\end{aligned}
$$

with

$$
V_{2}=e \beta\left(x-x_{k}\right)
$$

and

$$
V_{3}=e \beta x_{1} .
$$

$x_{k}$ is the first point and $x_{1}$ is the last point of the double barrier heterostructure. $e$ is the electron charge, $\beta$ gives the slope of the external field, and again $\chi$ is the characteristic theta function. The external field varies from zero to -18.4 $\times 10^{7} \mathrm{~V} / \mathrm{m}$, but all applied fields are between the orders of $-10^{7}--10^{8} \mathrm{~V} / \mathrm{m}$. For an average value of applied field, such as $\beta=-9.2 \times 10^{7} \mathrm{~V} / \mathrm{m}$, the beginning of the second barrier, $b_{2}$, is approximately $0.170 \mathrm{eV}$ lower than the beginning of the first barrier, $b_{1}$. For the same field, $V_{3}$ $=-0.268 \mathrm{eV}$. For the applied field of $\beta=-18.4$ $\times 10^{7} \mathrm{~V} / \mathrm{m}$, the beginning of $b_{2}$ has shifted down $0.340 \mathrm{eV}$ and $V_{3}$ is $-0.535 \mathrm{eV}$.

The barriers $b_{1}$ and $b_{2}$ are made of AlGaAs and have a height $.3 \mathrm{eV}$ and width $20 a_{0}$ with $a_{0}=0.529 \AA$. The outer wells, $w_{1}$ and $w_{3}$, are fabricated of GaAs and are $1100 a_{0}$ wide. The external barriers are constructed of doped GaAs and have a width of $B_{1}=B_{2}=440 a_{0}$ with a height $V$ $=0.9 \mathrm{eV}$. This is sufficient to completely confine the electron cloud, creating a closed system.

The initial wave packet is a Gaussian with a width $\sigma$ $=110 a_{0}$ and momentum $k_{0}$ that corresponds to the resonant energy of the unbiased double barrier, $E_{R}=.15 \mathrm{eV}$. Thus our initial wave function has the profile

$$
\Psi(x, 0)=\frac{1}{(\sigma \sqrt{\pi})^{1 / 2}} \exp \left\lfloor-\frac{1}{2}\left(\frac{x-x_{0}}{\sigma}\right)^{2}+i k_{0} x\right\rfloor .
$$

$x_{0}$ is placed in the middle of the well $w_{1}$ to insure that there is zero charge density initially inside the double barrier. It is worth elaborating here that Eq. (8) suggests that the wave function is normalized to unity, thus the problem is treated as a one electron problem. Our problem consists of a group of 


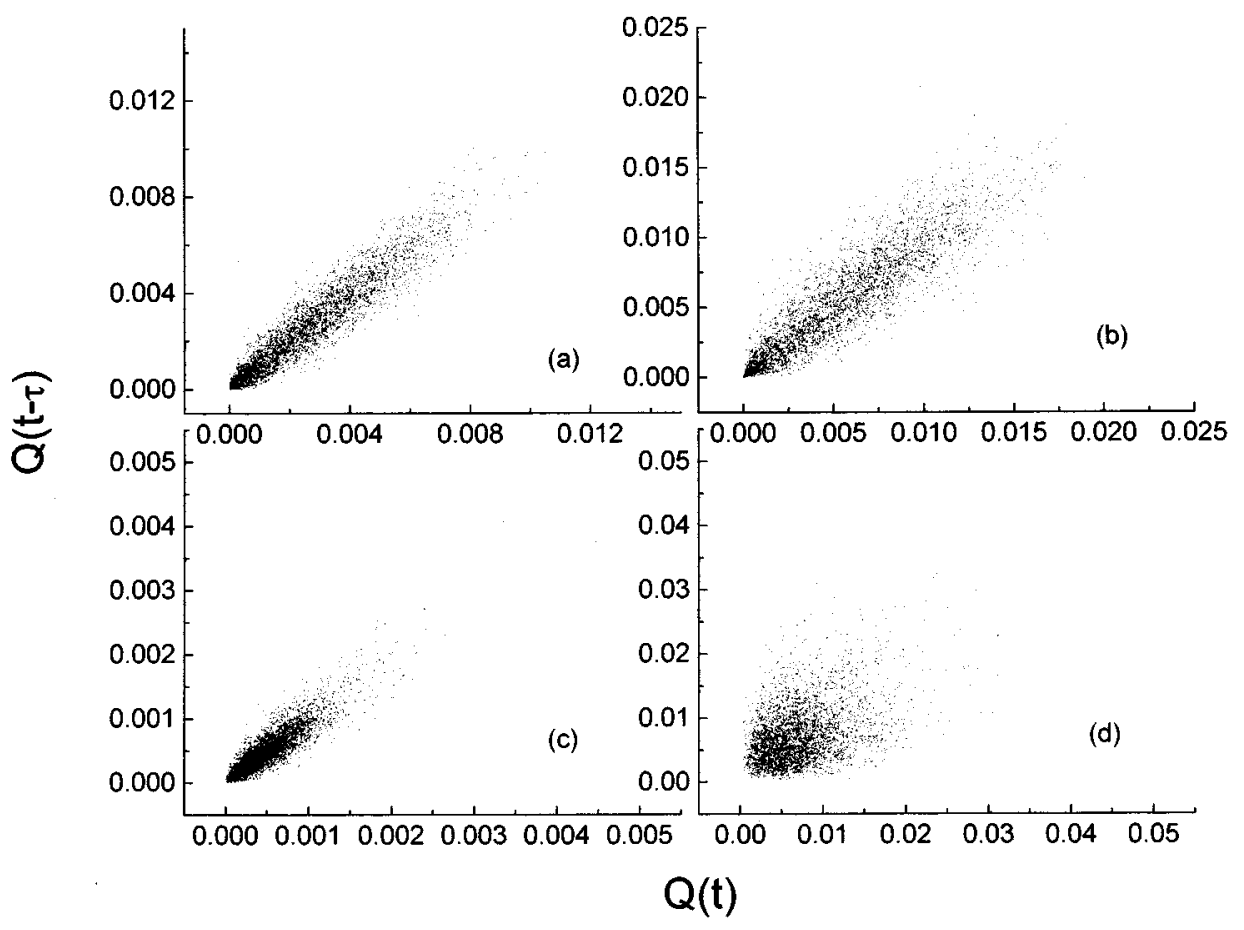

FIG. 2. Phase space plots for $\alpha=1.2$ and the dc fields of (a) $\beta=0$, (b) $\beta=-4.6 \times 10^{7}$, (c) $\beta=-9.2 \times 10^{7}$, (d) $\beta=-18.4 \times 10^{7} \mathrm{~V} / \mathrm{m}$.

electrons that are created in the large well and are launched towards the embedded layers of the heterostructure. An exact treatment of the problem is complicated due to the fact that this is a many body problem. Therefore the following assumptions are made:

(1) We assume a decoupling between the longitudinal and transverse degrees of freedom. This common assumption in tunneling problems reduces the equation to one dimension and allows the factorization of the wave function. Thus the experimental setup we have in mind places all the electrons in longitudinal symmetry.

(2) We assume the electrons in the group are uncorrelated. This corresponds to a wave function as the product of single particle states.

(3) Finally, we assume a single particle moving in the mean field of the rest of the electrons, resulting in a Hartreelike equation for our model. According to Theorem 5.7 of Ref. 7 by Spohn, this approximation is justified because we have a large number of electrons in our system.

The numerical technique used to solve the nonlinear, time dependent Schrödinger equation is given in detail in Ref. 4. This is solved independently for each electron which is treated as moving in a mean field dependent on the position of the rest of the electrons.

\section{NUMERICAL RESULTS}

The numerical integration over the central well, $w_{2}$, gives the amount of trapped charge $Q(t)$ vs time $t .{ }^{4,5}$ The time step used in computation is the atomic unit of time ( 1 a.u. $\left.=4.83 \times 10^{-17} \mathrm{~s}\right)$, and the output is every tenth time step.
Because the output is a single time series that is the composition of several factors, it is important to reduce the system to as small a state space as possible while still keeping the features of the system. Because the wavefunction, $\Psi(x, t)$, depends on the continuous space coordinate $x$, the variables span an infinite dimensional phase space. However, it has been shown that an infinite dimensional system might have a finite dimensional attractor. ${ }^{8}$ For our system, several parameters affect the behavior of the trapped charge. Among them are nonlinearity parameter, $\alpha$, mean incident energy, potential barrier height, well width, and applied dc electric field. In the interest of simplifying the dynamics of the system, we have fixed everything except $\alpha$ and the applied dc field, $\beta$. The reason for this is that $\alpha$ depends on the doping of the well and is not easily known upon the manufacturing of the heterostructure. It should, however, be measurable by matching the response of the electron build-up in the central well with the theoretical values. The applied dc field is also varied to study the effects of biasing on the motion of the electrons in the heterostructure.

\section{A. Phase space plot}

Because the output is a single time series, the phase space for the attractor was constructed by $Q(t)$ vs $Q(t-\tau)$ where $\tau$, the reconstruction time, is 100 a.u. This time was chosen both for continuity with the previous work, ${ }^{5}$ and because it is large enough to be measured experimentally using subpicosecond optical techniques described by Leo et al. ${ }^{9}$

Figures 2 and 3 show plots of the phase space in two dimensions. Figure 2 illustrates the interesting phenomena that occurs for low values of $\alpha$; in this case, $\alpha=1.2$. The applied fields are (a) zero, (b) $-4.6 \times 10^{7} \mathrm{~V} / \mathrm{m}$, (c) -9.2 $\times 10^{7} \mathrm{~V} / \mathrm{m}$, and (d) $-18.4 \times 10^{7} \mathrm{~V} / \mathrm{m}$. As the applied field 


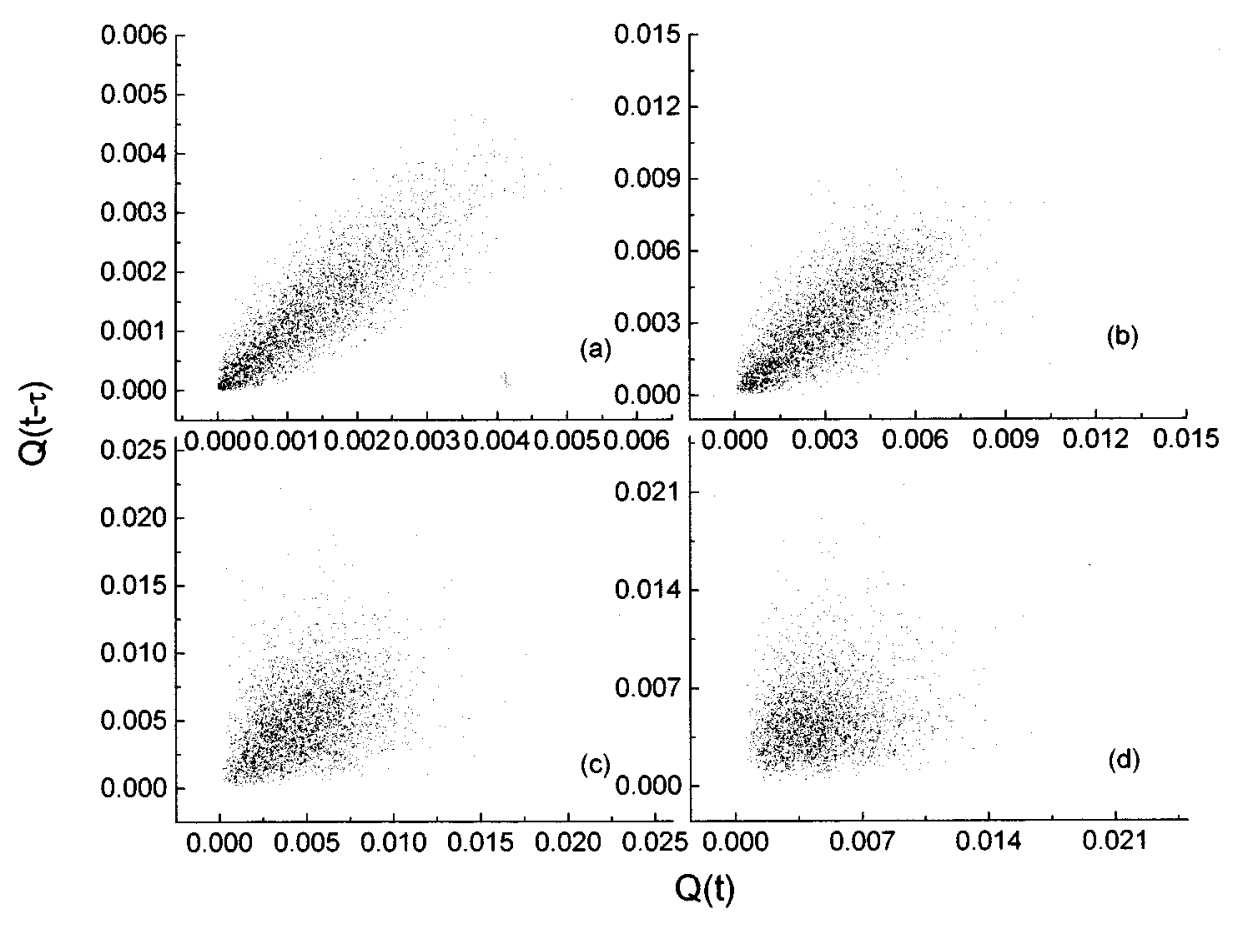

FIG. 3. Phase space plots for $\alpha=3$ and the dc fields of (a) $\beta=0$, (b) $\beta=-4.6 \times 10^{7}$, (c) $\beta=-9.2 \times 10^{7}$, (d) $\beta=-18.4 \times 10^{7} \mathrm{~V} / \mathrm{m}$.

increases, the phase space plot becomes spread out and mixes over a greater region. However, at $\beta=-9.2$ $\times 10^{7} \mathrm{~V} / \mathrm{m}$, the phase space plot actually contracts and becomes thinner. Yet as we keep increasing the field, the plot once again broadens until it becomes much more diffused than before as evident in Fig. 2(d). In contrast, Fig. 3 shows the phase space plot for $\alpha=3$. In this plot, the data continues to broaden, showing no signs of ever contracting back to the original form. Thus we already see an implied transition of behavior for the system between low and high values of $\alpha$. This aspect is further discussed in the last section of the article.

\section{B. Power spectrum}

The power spectrum of a periodic signal consists just of a series of peaks at the frequencies that make up the signal. Yet chaotic data, lacking any periodic behavior, should exhibit a continuum spectrum without any well defined peaks. This means the power spectrum can be used to distinguish between a signal that is just a composite of many frequencies, and one that may be chaotic. Although not a quantitative indicator of chaos, it can be used as an easy method of determining trends in data over a range of parameters.

The general conclusions from the phase space plots are reinforced by examining the power spectrums. For a low value, such as $\alpha=1.2$, there is a clear decrease in whiteness as shown in Fig. 4. Parts (a) and (b) for fields of $\beta=$ zero and $\beta=-4.6 \times 10^{7} \mathrm{~V} / \mathrm{m}$, respectively, show a clear whitening of the data that significantly decreases when $\beta=-9.2$ $\times 10^{7} \mathrm{~V} / \mathrm{m}$ as shown in Fig. 4(c). This is seen in the large dc spike and the large low frequency ac spikes, and the lack of power in the higher frequencies. Further, as the field is in- creased to $\beta=-18.4 \times 10^{7} \mathrm{~V} / \mathrm{m}$, the system becomes more chaotic and the frequencies are more evenly distributed in the higher region along with a dc spike and a low frequency spike. Also in agreement with the phase space plots is Fig. 5, the power spectrum for $\alpha=3$. It shows a continual increase in the broadening of the spectrum over the range of applied dc fields. As $\beta$ increases, the spectrum begins to resemble white noise, and for $\beta=-9.2 \times 10^{7} \mathrm{~V} / \mathrm{m}$, the only distinguishing feature is one large low frequency ac spike shown in Fig. 5(c). However, this feature quickly disappears, leaving a totally white spectrum when $\beta$ is increased to -18.4 $\times 10^{7} \mathrm{~V} / \mathrm{m}$ [Fig. 5(d)].

\section{Lyapunov exponent}

The measure of divergence or convergence of two nearby points in phase space is given by the Lyapunov exponent. Negative or zero exponents are features of fixed point and limit cycle systems. However, diverging trajectories exhibit positive Lyapunov exponents. In phase space, nearly identical states correspond to points in close proximity. An exponential divergence of these points magnify initial differences until the trajectories of the points behave quite differently. The Lyapunov exponent is a quantitative measure of the rate of this divergence. For a system to be defined as chaotic, it needs to have at least one positive exponent.

The equation for determining the Lyapunov exponent, $\lambda$, of a system is

$$
\lambda=\lim _{t \rightarrow \infty} \frac{1}{t} \log _{2} \frac{d(t)}{d(0)}
$$




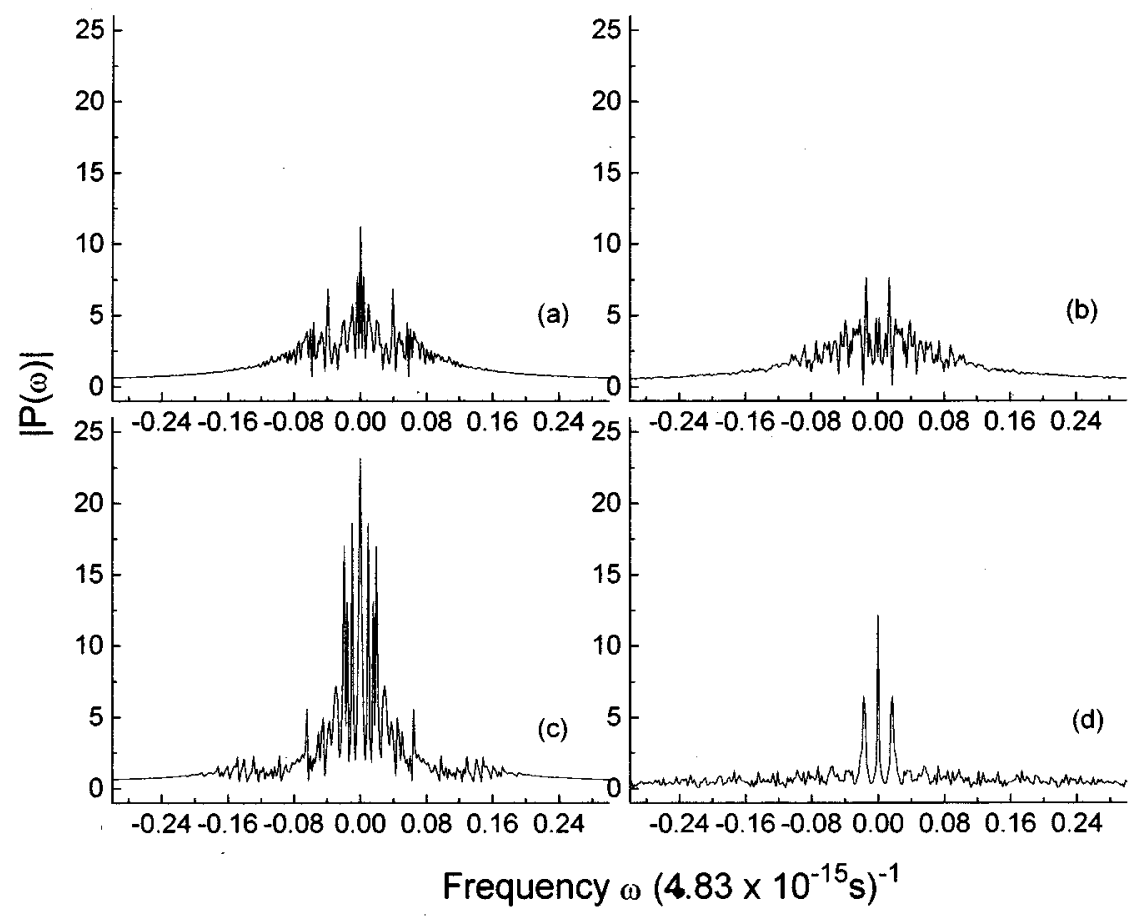

FIG. 4. Power spectrums for $\alpha=1.2$ and the dc fields of (a) $\beta=0$, (b) $\beta=-4.6 \times 10^{7}$, (c) $\beta=-9.2 \times 10^{7}$, (d) $\beta=-18.4 \times 10^{7} \mathrm{~V} / \mathrm{m}$.

In this equation, $t$ is the evolution time, $d(0)$ is the initial distance between two points on the normalized phase space diagram (Figs. 2 and 3), and $d(t)$ is the distance between the two points after both have been evolved along their trajectories by the evolution time $t .{ }^{10}$ For our system, these distances on the phase space are given by the formula

$$
\begin{aligned}
d(0)= & \left\{\left[Q\left(t_{2}\right)-Q\left(t_{1}\right)\right]^{2}+\left[Q\left(t_{2}-\tau\right)-Q\left(t_{1}-\tau\right)\right]^{2}\right\}^{1 / 2} \\
d(t)= & \left\{\left[Q\left(t_{2}+t\right)-Q\left(t_{1}+t\right)\right]^{2}+\left[Q\left(t_{2}+t-\tau\right)\right.\right. \\
& \left.\left.-Q\left(t_{1}+t-\tau\right)\right]^{2}\right\}^{1 / 2} .
\end{aligned}
$$

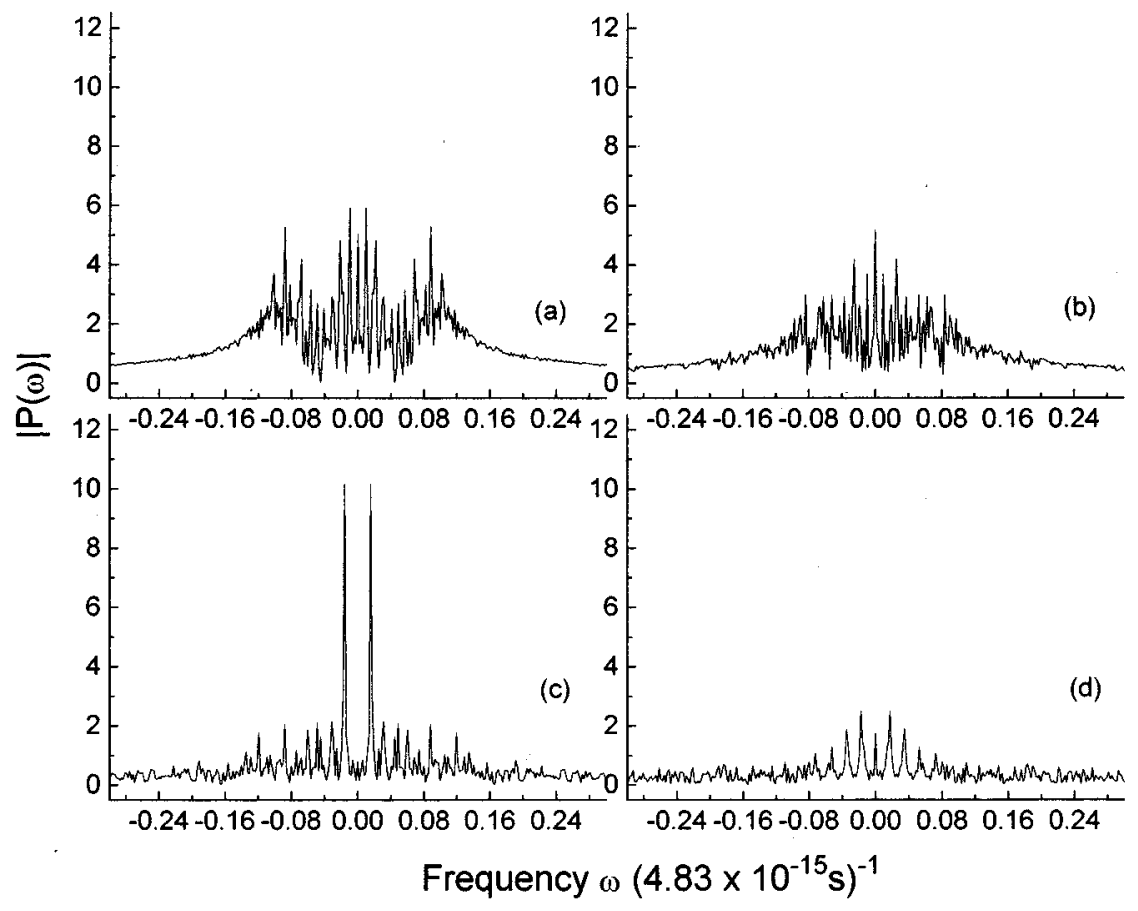

FIG. 5. Power spectrums for $\alpha=3$ and the dc fields of (a) $\beta=0$, (b) $\beta=-4.6 \times 10^{7}$, (c) $\beta=-9.2 \times 10^{7}$, and (d) $\beta=-18.4 \times 10^{7} \mathrm{~V} / \mathrm{m}$. 


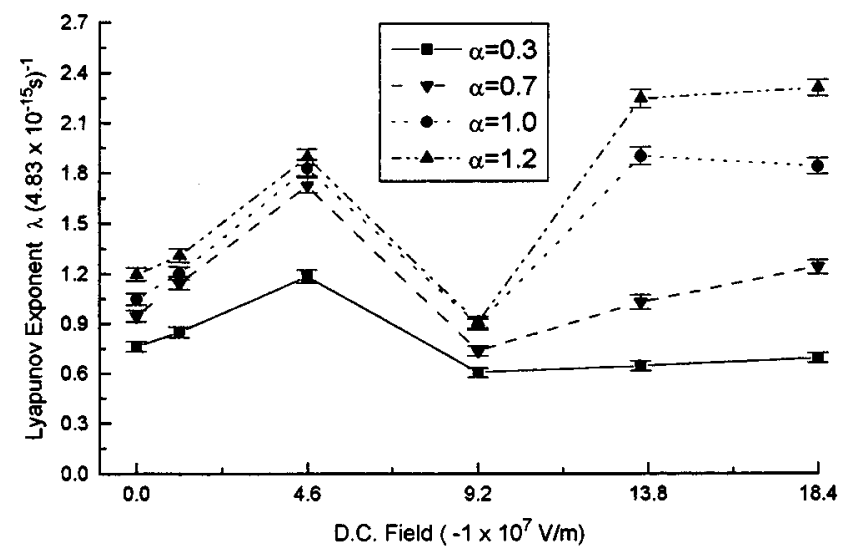

FIG. 6. Lyapunov exponent vs applied dc field for several values of $\alpha$ $\leqslant 1.2$.

Here $\tau$ is the reconstruction time of the attractor in phase space, and $t_{1}$ and $t_{2}$ are initial points in the time series chosen such that $d(0)$ is as small as possible without going below the noise scale $\left(\sim 10^{-4}\right)$.

The distance between two close points, $t_{1}$ and $t_{2}$, is calculated. After the points are evolved by the time, $t$, the distance is recalculated. The Lyapunov exponent is then defined as the $\log$ of the ratio of the distances scaled by the time evolved. ${ }^{11}$ Because numerically we are dealing with discrete points instead of a continuum, it is necessary to calculate this value as many times as possible to obtain an average value for the exponent. In doing this, the first point, $t_{1}$, is kept and evolved through the entire data set. For every step, the second point, $t_{2}$, is selected such that $d(0)$ is small. The Lyapunov exponent is then calculated for a given evolution time, $t$. To start the next step, the evolved point $t_{1}$ $+t$ is renamed as $t_{1}$. The point $t_{2}+t$ is discarded and a new $t_{2}$ is chosen to satisfy the conditions on $d(0)$. There is a Lyapunov exponent for every dimension of the phase space, but since only one positive exponent is necessary to show chaos, only the largest exponent is calculated. ${ }^{10}$

Figure 6 gives quantitative results backing up the trends shown in the phase space plots and power spectrums. For values of $\alpha$ near to and less than 1.2, we see a large drop in the chaos of the system at $\beta=-9.2 \times 10^{7} \mathrm{~V} / \mathrm{m}$. This trend persists all the way down to extremely low values of $\alpha$, where the Lyapunov exponent is also low for higher values of $\beta$. However, at $\alpha=1.376$, there is a first-order type transition where $\lambda$ jumps to high values, eliminating the dip as seen in Fig. 7. Figure 8 clearly shows this transition for the range of $\alpha$ values between 1.2 and 1.5 for the applied field value of $\beta=-9.2 \times 10^{7} \mathrm{~V} / \mathrm{m}$. In addition, Fig. 8 shows the progression of the average nonlinear potential, $\alpha\langle Q(t)\rangle$, as a function of $\alpha$. It shows a first-order type transition that corresponds exactly with the jump in $\lambda$. This sudden increase in potential is due to a large increase in the mean charge trapped in the well caused by resonance effects in the heterostructure.

The behavior of the mean charge in the well is shown in Fig. 9 for all the fields. The very low applied fields and zero field cases exhibit only a drop in the mean charge as a function of $\alpha$. For values of $\beta>-4.6 \times 10^{7} \mathrm{~V} / \mathrm{m}$, the mean

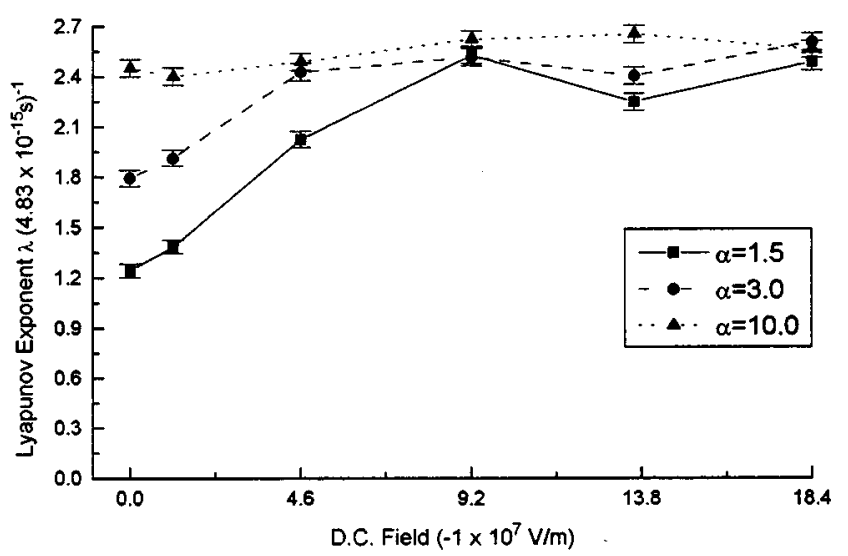

FIG. 7. Lyapunov exponent vs applied dc field for several values of $\alpha$ $\geqslant 1.5$.

charge stays low for a range of $\alpha$ values before a sharp increase to a peak and a slow tapering off afterwards. This is due to the resonance build-up in the heterostructure as a function of $\alpha$, and it is most prominent for $\beta=-9.2$ $\times 10^{7} \mathrm{~V} / \mathrm{m}$ whose mean charge stays the lowest for the longest range of $\alpha$. This shows that chaos in the system stays correlated to the mean charge for the value of $\beta=-9.2$ $\times 10^{7} \mathrm{~V} / \mathrm{m}$. In both the mean charge and in the Lyapunov exponent, the values stay fairly low until $\alpha=1.376$ when both values go through their transition. Both values stay large after that point (Figs. 8 and 9). In addition, for large values of $\alpha$ and for all fields, the Lyapunov exponents converge to similar values around $\lambda=2.5$ as shown in Fig. 7. The correlation of the mean charge and $\lambda$ is demonstrated again in Fig. 9 by the settling down to a similar value of mean charge for all values of $\beta$ and large values of $\alpha$. In addition for $\alpha=10$, a systematic increase in the mean charge as a function of $\beta$ is noticed.

\section{DISCUSSION}

There is a general trend in the biased heterostructure that as $\beta$ increases for a particular value of $\alpha$, the chaos in the

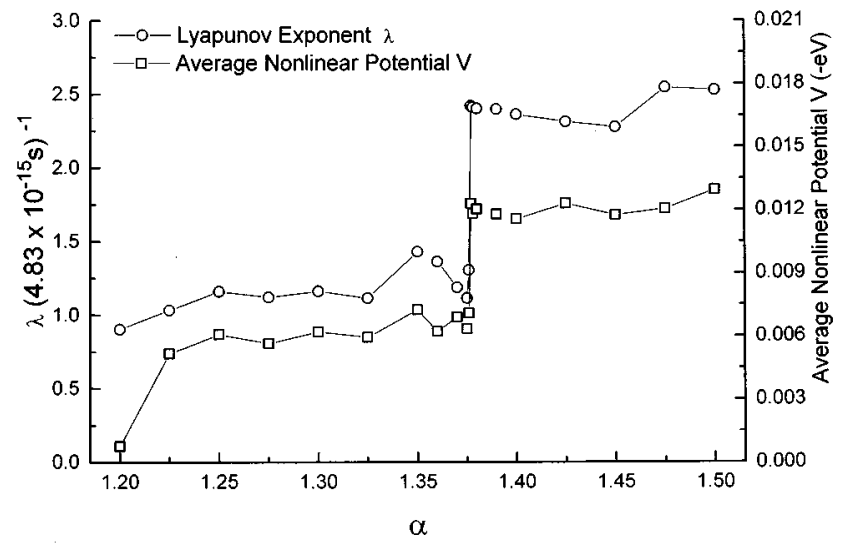

FIG. 8. Lyapunov exponent $\lambda$ vs $\alpha$ and average nonlinear potential $V$ vs $\alpha$ for the applied dc field of $\beta=-9.2 \times 10^{7} \mathrm{~V} / \mathrm{m}$. 


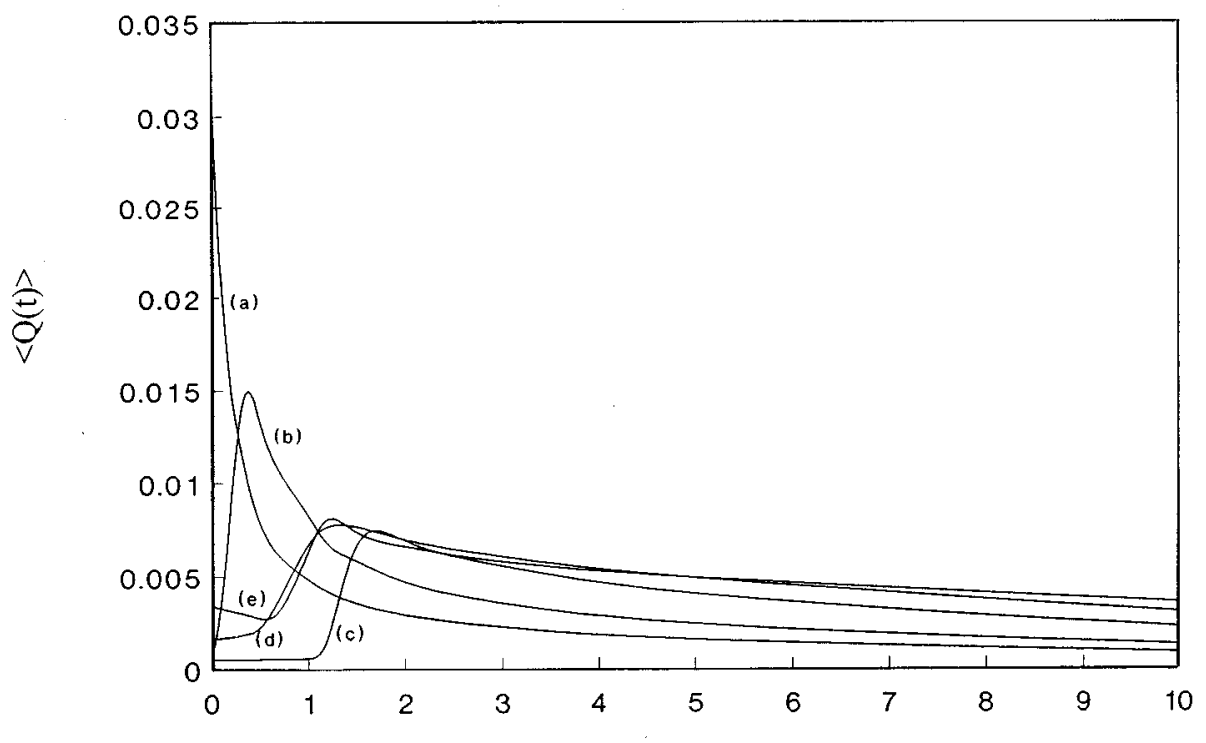

$\alpha$

FIG. 9. Mean charge vs $\alpha$ for several applied dc fields of (a) $\beta=-1.15 \times 10^{7}$, (b) $\beta=-4.6 \times 10^{7}$, (c) $\beta=-9.2 \times 10^{7}$, (d) $\beta=-13.6 \times 10^{7}$, and (e) $\beta$ $=-18.4 \times 10^{7} \mathrm{~V} / \mathrm{m}$.

system increases. The mixing that occurs in the phase space is shown for $\alpha=3$ in Fig. 3 and also in the near even distribution of frequencies in the power spectrum (Fig. 5). It is also shown in three of the four fields in the phase space for $\alpha=1.2$ (Fig. 2) and in the same three out of four fields for Fig. 4 which is the power spectrum. As $\beta$ increases, the well becomes increasingly off resonant with the mean energy of the initial electron packet. As far as the quantitative results go, the biased heterostructure can be divided into three cases. Low values of $\alpha(\leqslant 1.2)$ show an initial increase in $\lambda$, followed by a drop in value and subsequent regrowth of the Lyapunov exponent as illustrated in Fig. 6. The intermediate values of nonlinearity, $1.2<\alpha<10$ show a steady increase in the Lyapunov exponent to a saturation point around $\lambda \approx 2.5$ (Fig. 7). For large values of $\alpha$, the nonlinear effects dominate the heterostructure, and chaos emerges independent of the applied field as illustrated by Fig. 7 .

Let us first examine the behavior of the heterostructure as we go from low values of $\alpha$ to high values for the applied dc field of $\beta=-9.2 \times 10^{7} \mathrm{~V} / \mathrm{m}$. The applied field slants the well down as shown in Fig. 1(b), and this lowers the energy levels inside the well. At $\beta=-9.2 \times 10^{7} \mathrm{~V} / \mathrm{m}$, the lowered energy levels provide for an off-resonant situation with respect to the mean kinetic energy of the pulse. This results in the low mean charge build-up for values of $\alpha \leqslant 1.2$ (Fig. 9), and it translates into weak chaos for those values (Fig. 6). The effect of the nonlinear term $\alpha$ is to broaden the energy levels in the well up toward the mean kinetic energy of the Gaussian packet. The values of $\alpha$ that cause the peak in the mean charge for a specific $\beta$ correspond to the case when the mean energy of the level is in resonance with the average kinetic energy of the incident electron packet. ${ }^{4}$ For a sufficiently strong nonlinearity, the energy levels get broadened enough that there is sufficient charge trapped in the well to raise the Lyapunov exponent drastically as shown in Figs. 7 and 8. This relation between nonlinear potential and mean charge build-up in the well is shown explicitly as functions of $\alpha$ in Fig. 10. This figure plots the average nonlinear potential $\alpha\langle Q(t)\rangle$ and the average charge trapped for four different values of $\beta$. For sufficiently high values of $\alpha$, fluctuations around the average value of charge trapped in the double barrier eventually push the nonlinear potential into a resonant situation with respect to the mean kinetic energy of the electrons. Once on resonance, the increase in the amount of charge trapped in the well keeps the energy level of the well at resonance. With the increase in trapped charge, there is a greater variance in the nonlinear potential due to its higher average, and therefore there is an increase in $\lambda$. Thus, as shown in Fig. 6, for values of $\alpha \leqslant 1$.2, field effects dominate and there is a strong regular oscillation in the charge in the well as shown in the large ac peaks in the low frequency range in Fig. 4(c). The tailing behavior of the mean charge in Fig. 9 is due to the fact that for large $\alpha$ the energy levels have become so broadened that the mean energy of the level is once again off resonant with the initial kinetic energy of the electron packet. ${ }^{4}$ However, by this point the nonlinear effects dominate the system, and there is no decrease in Lyapunov exponent corresponding to the slow drop in mean charge.

The interesting phenomena in this system for the applied dc field of $\beta=-9.2 \times 10^{7} \mathrm{~V} / \mathrm{m}$ occurs for $\alpha$ values between $\alpha=1.2$ and $\alpha=1.5$. As shown in Fig. 8, when $\alpha$ increases, we have a first order type transition in $\lambda$ and in the average nonlinear potential, $V$. To see how the resonance effects cause this transition, we examine the amount of charge, $Q(t)$, in the central well as a function of time as shown in Fig. 11. This clearly shows a transition in the raw data from 


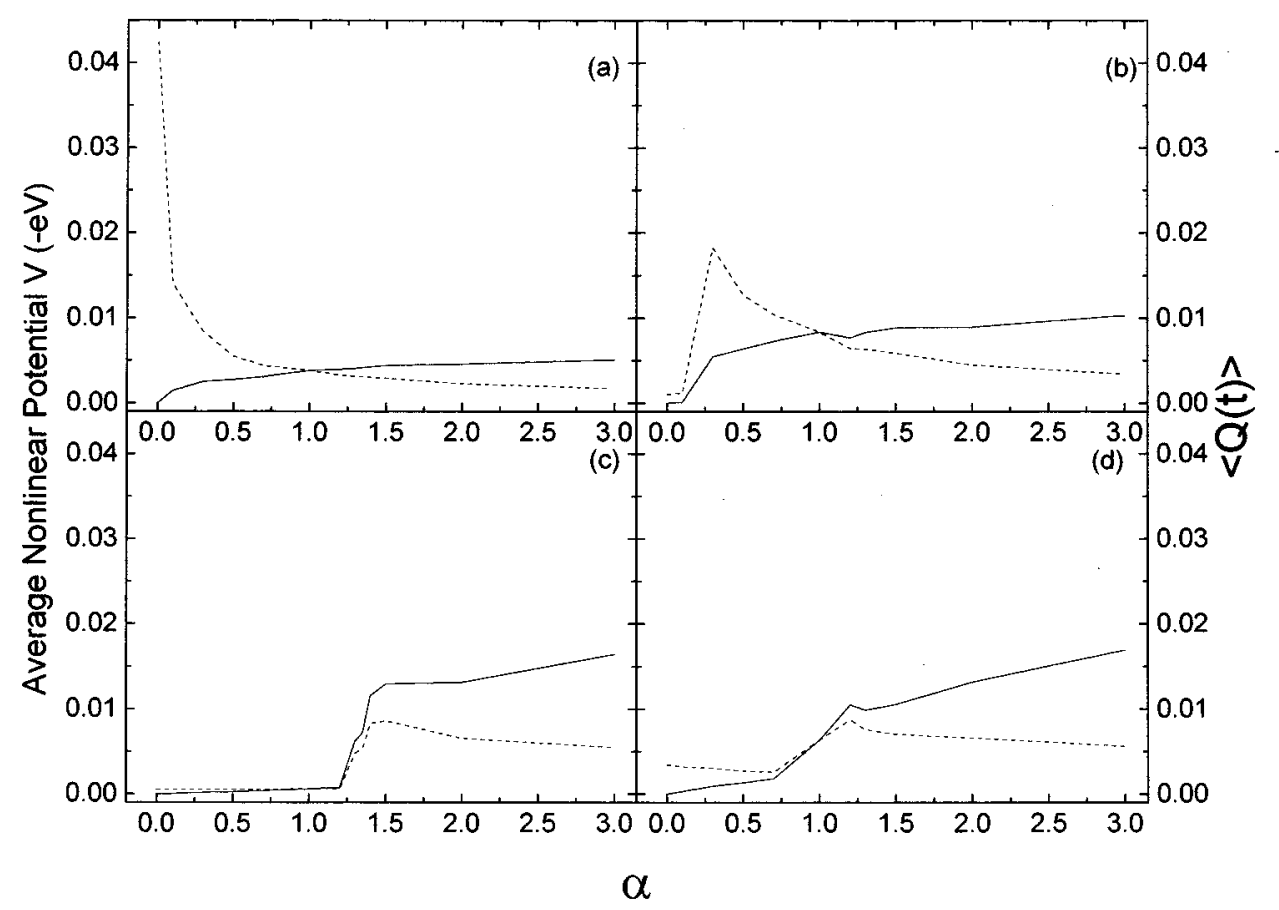

FIG. 10. Average nonlinear potential vs $\alpha$ (solid line) and mean charge vs $\alpha$ (dashed line) for the applied dc fields of (a) $\beta=0$, (b) $\beta=-4.6 \times 10^{7}$, (c) $\beta$ $=-9.2 \times 10^{7}$, and (d) $\beta=-18.4 \times 10^{7} \mathrm{~V} / \mathrm{m}$.

a nonresonant to a resonant state for each of the $\alpha$ values: $1.25,1.376,1.377$, and 1.475 . For $\alpha \leqslant 1.2$, this transition does not occur, while for values of $\alpha \geqslant 1.5$, this transition occurs almost immediately. The transition to resonance occurs when $Q(t) \geqslant .01$, which corresponds to $1 \%$ of the total charge in the three well heterostructure. It is noted that for values of $\alpha \leqslant 1.376$, this transition to resonance happens late, for times $t>350000$ a.u. But for higher values of $\alpha$, the transition occurs for $t<100000$ a.u. The sudden decrease in the amount of time to resonance in the well causes the first order type transition in the average nonlinear potential and in the Lyapunov exponent. Despite the decrease in mean charge

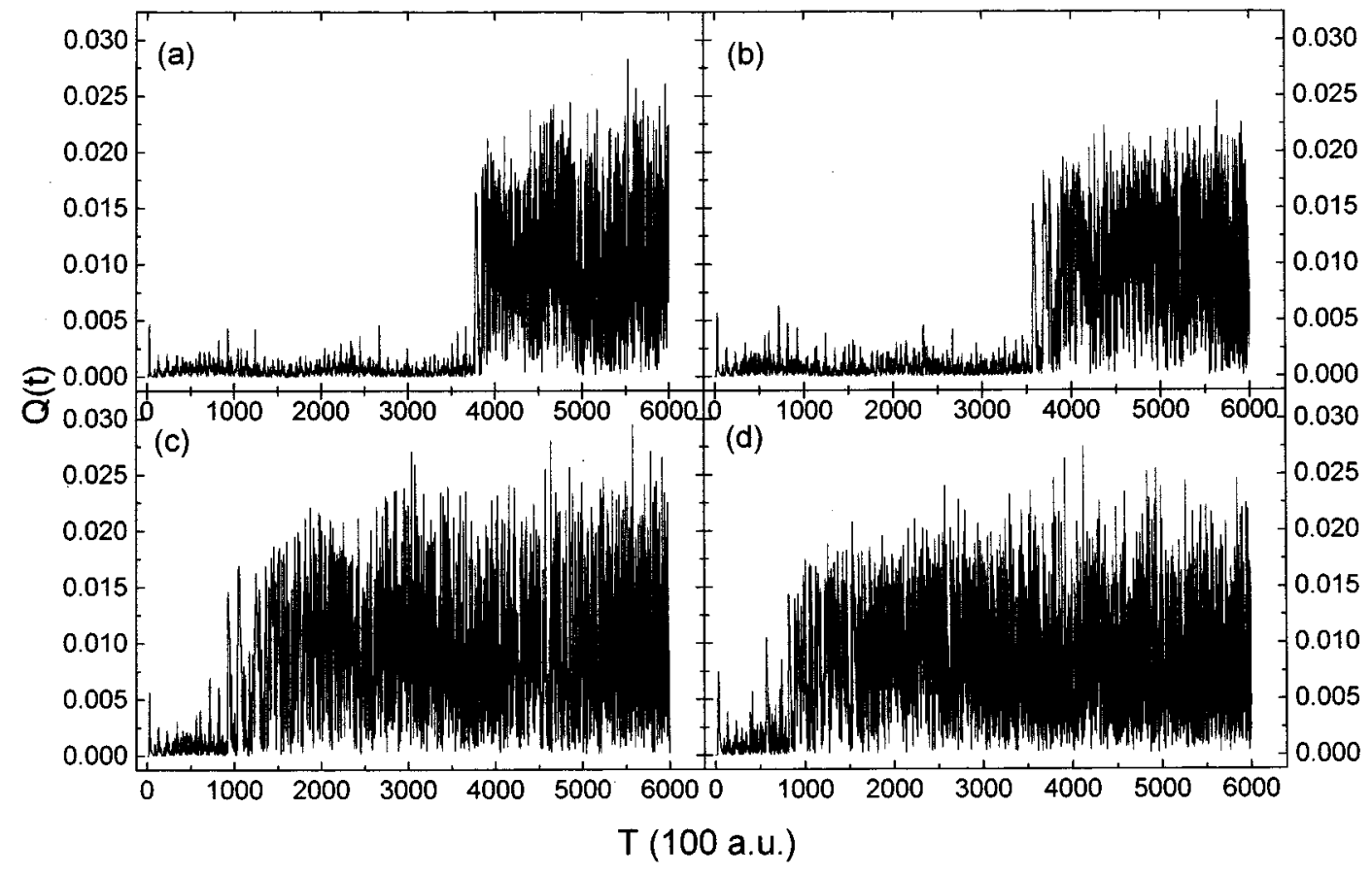

FIG. 11. $Q(t)$ vs $T$ for the applied dc field of $\beta=-9.2 \times 10^{7} \mathrm{~V} / \mathrm{m}$ and nonlinearity coefficients (a) $\alpha=1.25$, (b) $\alpha=1.376$, (c) $\alpha=1.377$, (d) $\alpha=1.475$. 
for $\alpha \geqslant 1.5$ as shown in Fig. 10(c), the level broadening keeps $Q(t)$ on resonance although the resonance is weaker due to the increased repulsive potential of $\alpha\langle Q(t)\rangle$.

As stated previously, quantum heterostructures are biased with strong electric fields to increase the operating frequency of the device. Under an external field of $\beta=-9.2$ $\times 10^{7} \mathrm{~V} / \mathrm{m}$, the characteristic time, $\tau$, of the heterostructure is halved. ${ }^{4}$ This corresponds to the field under which the Lyapunov exponent drops significantly for low $\alpha$. It is also the field that has low charge build-up for the largest range of $\alpha$. This makes the system useful as an electronic device since low charge build-up and weak chaos implies a strong regular current through the device. This is backed up by the short response time that leads to a high operating frequency. These features can be seen in Fig. 4(c) where the power spectrum indicates a strong periodic build-up and release of charge through the heterostructure.

The model system described in this article can be constructed and tested by choosing materials for the barriers $b_{1}, b_{2}$, and the thin well, $w_{2}$, to be AlGaAs and GaAs, respectively. The wide wells, $w_{1}$ and $w_{3}$, are to be constructed of doped GaAs. The electron wave packet in the wide well, $w_{1}$, can be created using an ultrashort laser pulse. Excitation with a spectrally broad pulse encompassing many optical transitions of the system can create a wave packet. The time evolution of the wave packet can be investigated by using delayed probe pulses and by time resolved four-wave mixing. ${ }^{8}$ In this manner, the oscillating charge trapped in the biased heterostructure can be measured experimentally with subpicosecond optical techniques.

\section{ACKNOWLEDGMENT}

A. Jason McNary was supported by a fellowship from Board of Regents Support Fund (BoRSF). The authors gratefully thank Pat Robbert for her assistance in running data sets so that this research could be completed in a timely manner. We are also grateful for her critical reading of the manuscript.

${ }^{1}$ F. Capasso and S. Datta, Phys. Today 43, 74 (1990).

${ }^{2}$ S. Lury, Appl. Phys. Lett. 47, 490 (1985).

${ }^{3}$ A. B. Fowler, U.S. Patent No. 45503320 (1985); S. Datta, M. R. Melloch, S. Bandyopadhyay, and M. S. Lundstrom, Appl. Phys. Lett. 48, 487 (1986).

${ }^{4}$ A. Rosa, A. Puri, J. E. Murphy, and T. Odagaki, J. Appl. Phys. 75, 5196 (1994).

${ }^{5}$ A. J. McNary and A. Puri, J. Appl. Phys. 80, 247 (1996).

${ }^{6}$ G. Jona-Lasinio, C. Presilla, and F. Capasso, Phys. Rev. Lett. 68, 2269 (1992).

${ }^{7}$ H. Spohn, Rev. Mod. Phys. 53, 569 (1980).

${ }^{8}$ J. D. Farmer, Physica D 4, 366 (1982).

${ }^{9}$ K. Leo, I. Shah, E. O. Göbel, T. C. Damen, S. Schmitt-Rink, W. Schäfer, and K. Köhler, Phys. Rev. Lett. 66, 201 (1991).

${ }^{10}$ A. Wolf, J. B. Swift, H. L. Swinney, and J. A. Vastano, Physica D 16, 285 (1985).

${ }^{11}$ J. L. McCauley, in Chaos, Dynamics, and Fractals-an Algorithmic Approach to Deterministic Chaos (Cambridge University Press, New York, 1993). 